
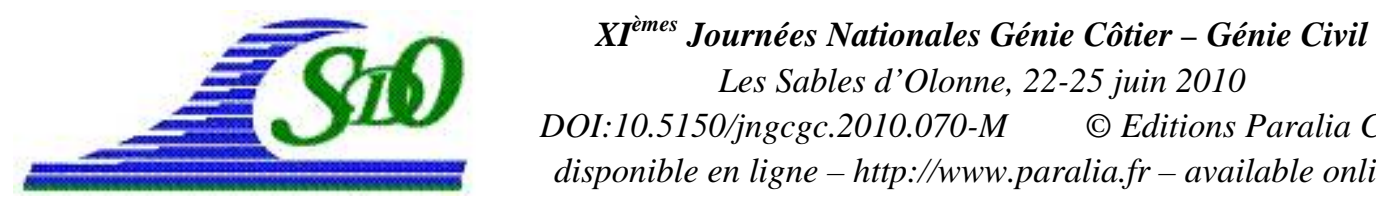

\title{
Evolution de la ligne de côte du littoral de Sidi-Fredj (Algérie)
}

\section{Khoudir MEZOUAR ${ }^{1}$, Romeo CIORTAN ${ }^{2}$, Mohamed Amine BOUKHEMACHA ${ }^{1}$}

\author{
1. Université Techniques de Construction de Bucarest, B-dul Lacul Tei, Nr.124, \\ 020396 Bucuresti, Romania. \\ mezouarkhoudir@yahoo.fr ; boukhemacha.amine@hotmail.com \\ 2. IPTANA S.A. et Université d’Ovidiu Constanta, str Unirii, Nr 22 B, Constanta, \\ Romania. \\ ciortanromeo@yahoo.com
}

\section{Résumé :}

L'analyse diachronique des photographies aériennes montre que l'érosion de la plage du littoral sableux de Sidi-Fredj s'est produite à des vitesses variables entre 1972 et 2008. La période de 1972-1980 enregistre une érosion généralisée avec un taux moyen de 4 m/an dans la partie Est. La période de 1980-1993 enregistre un rééquilibrage avec la l'apparition de deux secteurs, le premier en érosion avec un taux moyen de $-4.64 \mathrm{~m} / \mathrm{an}$, le deuxième en engraissement au taux moyen de $1.85 \mathrm{~m} / \mathrm{an}$. En effet, à cause de son isolement des zones d'alimentation en sable, la plage de la baie de Sidi-Fredj évolue en système fermé. D’une part, la construction du port a entravé le transit littoral engendré par la houle ouest et, d'autre part, l'aménagement du bassin de l'oued Beni-Messous a immobilisé ses apports terrigènes qui ne contribuent donc plus à l'apport solide actuel. Enfin le courant de dérive vers l'Est, qui redistribue les venues sédimentaires, a été interrompu par la construction d'un système de défense. Les apports sont désormais immobilisés entre ces trois épis et ne contribuent plus à la dynamique sédimentaire, ce qui aggrave ainsi l'appauvrissement du stock sédimentaire mis en jeu. La plage Est, n’étant plus alimentée par les sédiments acheminés par la dérive Est comme auparavant, verra sa vulnérabilité augmenter progressivement. Sa cicatrisation à la suite des tempêtes successives se fera de plus en plus difficilement. En conclusion, la reconstitution du système côtier naturel et le suivi des aménagements successifs de la baie montrent que l'anthropisation du littoral a déséquilibré le système de manière importante et sans doute irréversible.

\section{Mots-clés :}

Littoral - Sidi-Fredj - Dynamique sédimentaire - Recul de rivage - Plage

\section{Communication non présentée}


Thème 4 - Vulnérabilité du littoral 
XI ${ }^{\text {èmes }}$ Journées Nationales Génie Côtier - Génie Civil

Les Sables d'Olonne, 22-25 juin 2010 
Thème 4 - Vulnérabilité du littoral 
XI ${ }^{\text {èmes }}$ Journées Nationales Génie Côtier - Génie Civil

Les Sables d'Olonne, 22-25 juin 2010 
Thème 4 - Vulnérabilité du littoral 
XI $I^{\text {èmes }}$ Journées Nationales Génie Côtier - Génie Civil

Les Sables d'Olonne, 22-25 juin 2010 
Thème 4 - Vulnérabilité du littoral 